\title{
Anti B-cell maturation antigen CAR T-cell and antibody drug conjugate therapy for heavily pretreated relapsed and refractory multiple myeloma
}

\author{
A summary from the Institute for Clinical and Economic Review's Midwest Comparative \\ Effectiveness Public Advisory Council
}

Molly Beinfeld, MPH; Sei Lee, MD, MAS; Brett McQueen, PhD; Noemi Fluetsch, MPH; Steven D Pearson, MD, MSc; and Daniel A Ollendorf, PhD

Multiple myeloma (MM) is a hematologic cancer of plasma cells, primarily affecting older individuals. Approximately 32,000 cases are diagnosed each year, and 150,000 people are living with $\mathrm{MM}$ in the United States. ${ }^{1}$ Black Americans appear to have approximately twice the risk of developing MM compared with White Americans. ${ }^{2}$ Direct medical costs of treating $\mathrm{MM}$ are substantial; in a recent claims analysis, average costs exceeded \$250,000 over a 21-month period, and the majority of these costs were medication related. ${ }^{3}$

Current treatment of MM typically includes 3 general classes of drugs: immunomodulatory agents, proteasome inhibitors, and antiCD38 monoclonal antibodies. ${ }^{4}$ Unfortunately, most patients eventually relapse; patients with relapsed or refractory MM (RRMM) often cycle through different combinations of available agents. When a patient's disease is no longer responding to agents in each of these classes, the disease is referred to as "triple-class refractory" MM (TCRMM). ${ }^{4}$ Response rates to existing, later-line therapies are relatively low, underscoring the need for new treatment options in this heavily pretreated population.

The Institute for Clinical and Economic Review (ICER) conducted a systematic literature review and costeffectiveness analysis to evaluate the health and economic outcomes of 3 new treatments targeting the B-cell maturation antigen (BCMA) for heavily pretreated patients with RRMM. BCMA is preferentially expressed on plasma cells, making it an attractive therapeutic target for MM. Belantamab mafodotin blmf (Blenrep, GlaxoSmithKline) is an antibody drug conjugate, with a monoclonal antibody to BCMA linked to a cytotoxic drug. Belantamab was studied in patients with heavily pretreated (6-7 previous lines of therapy) TCRMM (majority quad- and penta-refractory, usually defined as refractory to 4 or 5 agents across all 3 drug classes previously mentioned). Idecabtagene vicleucel (ide-cel, Abecma, Bristol Myers Squibb and bluebird bio) and ciltacabtagene autoleucel (cilta-cel, Janssen and Legend biotech) are chimeric antigen receptor (CAR) T-cell therapies, involving engineering a patient's own T cells to target BCMA.

\section{Author affiliations}

Molly Beinfeld, MPH; Noemi Fluetsch, $\mathrm{MPH}$; and Steven D Pearson, MD, MSc, Institute for Clinical and Economic Review, Boston, MA. Sei Lee, MD, MAS, University of California, San Francisco; Brett McQueen, PhD, Skaggs School of Pharmacy and Pharmaceutical Sciences, University of Colorado Anschutz Medical Campus, Aurora; and Daniel A Ollendorf, $\mathrm{PhD}$, Center for the Evaluation of Value and Risk in Health, Tufts Medical Center, Boston, MA.

AUTHOR CORRESPONDENCE:

Molly Beinfeld, mbeinfeld@icer.org

J Manag Care Spec Pharm. 2021;27(9):1315-20

Copyright $\odot 2021$, Academy of Managed Care Pharmacy. All rights reserved.

Ide-cel and cilta-cel were studied in patients who were mostly TCRMM (majority triple- or quad-refractory patients). Belantamab was approved by the US Food and Drug Administration (FDA) for RRMM in August 2020; idecel was approved in March 2021; and 


\begin{tabular}{|c|c|c|c|c|c|}
\hline Intervention & Trial & $\begin{array}{l}\text { Median follow-up } \\
\text { duration }\end{array}$ & $\begin{array}{l}\text { As-treated PFS, } \\
\text { median months } \\
(95 \% \mathrm{Cl})\end{array}$ & $\begin{array}{l}\text { As-treated OS, } \\
\text { median months } \\
(95 \% \mathrm{Cl})\end{array}$ & $\begin{array}{c}\text { ITT ORR, } \\
\text { n (\%); [95\% CI] }\end{array}$ \\
\hline Ide-cel & $\begin{array}{l}\operatorname{KarMMa}^{5,7} \\
(\mathrm{~N}=149)^{\mathrm{a}}\end{array}$ & 13.3 months $^{\mathrm{b}}$ & $8.8(5.6-11.6)^{\mathrm{b}}$ & $19.4(18.2, \mathrm{NE})^{\mathrm{b}}$ & 94 (63.0); [NR] \\
\hline Cilta-cel & $\begin{array}{l}\text { CARTITUDE-13,8, } \\
(\mathrm{N}=126)^{\mathrm{a}}\end{array}$ & 18 months $^{\mathrm{b}}$ & $\begin{array}{l}\text { Not reached at } \\
18 \text { months }^{\mathrm{b}}\end{array}$ & $\begin{array}{l}\text { Not reached at } \\
18 \text { months }^{b}\end{array}$ & 95 (75.0); [NR] \\
\hline Belantamab & $\begin{array}{l}\text { DREAMM- } 2^{6} \\
(\mathrm{~N}=97)^{\mathrm{a}, \mathrm{c}}\end{array}$ & 13 months & $2.8(1.6-3.6)^{b}$ & $\begin{array}{l}13.7 \\
(9.9, \text { not reached })^{a}\end{array}$ & $\begin{array}{l}31 \text { (32.0); } \\
\text { [97.5\% Cl: 21.7-43.6] }\end{array}$ \\
\hline \multicolumn{6}{|c|}{$\begin{array}{l}\text { Intention to treat. } \\
\text { 'Median follow-up duration, PFS and OS for KarMMa and CARTITUDE-1 are based on the as-treated population. } \\
\text { '2.5 mg/kg arm only. } \\
\text { ITT=intention to treat; } N E=\text { not estimable; } N R=\text { not reported; } O R R=\text { overall response rate; } O S=\text { overall survival; } P F S=\text { progression-free survival. }\end{array}$} \\
\hline
\end{tabular}

cilta-cel was granted fast track designation and has a Prescription Drug User Fee Act (PDUFA) target action date of November 29, 2021.

Complete details of ICER's systematic literature search and protocol, as well as the methodology and model structure for the economic evaluation, are available on ICER's website at https://icer.org/assessment/multiplemyeloma-2021/. In this review, we present the summary of our findings and highlights of the policy discussion with key stakeholders held at a public meeting of the Midwest Comparative Effectiveness Public Advisory Council on April 16, 2021. In addition, new data for cilta-cel has become available since that meeting and is highlighted here.

\section{Summary of Findings}

\section{CLINICAL EFFECTIVENESS}

The systematic literature review identified only 2 openlabel, single arm phase 1/2 studies of the CAR-Ts (one each for ide-cel and cilta-cel) and 1 open-label phase 2 study of belantamab, making indirect comparisons of relative effectiveness impossible. At the time of the review, only the pivotal trials of ide-cel and belantamab had been published. ${ }^{5,6}$ Our review also evaluated information from conference abstracts, regulatory documents, and data submitted by the manufacturers.

The pivotal trials of ide-cel and cilta-cel included similar distributions of baseline characteristics (such as age and previous lines of therapy); however, the ide-cel trial included more patients with extramedullary disease (a marker of more aggressive disease) and high-risk cytogenetics. ${ }^{3,5}$ The pivotal trial of belantamab included patients who were older, had undergone more pretreatment, and were more likely to have high-risk cytogenetics compared with the patient populations in the CAR-T trials. ${ }^{6}$ A key difference between the pivotal CAR-T therapy trials and the belantamab trial was the approach for inclusion in the outcomes analysis. The CAR-T trials only included patients who were infused in the analysis in an "as-treated" approach, whereas the belantamab trial reports on the full intentionto-treat (ITT) population. At the time of this publication, only interim, unpublished data on cilta-cel was available. ${ }^{3,5,6}$

In the pivotal trial of ide-cel, with a median follow-up of 13.3 months, as-treated median progression-free survival (PFS) and overall survival (OS) were 8.8 months and 19.4 months, respectively. Using an ITT approach, 94 of 149 enrolled patients (63\%) achieved an overall response to treatment (Table 1). Twelve-month OS and PFS were $78 \%$ and $38 \%$, respectively. ${ }^{5,7}$

Subsequent to the publication of the final ICER report, updated data from the pivotal trial of cilta-cel were released. ${ }^{8}$ With a median follow-up of 18 months, median PFS and OS had not been reached. Ninety-five of 126 enrolled patients (75\%) achieved an overall response (Table 1). ${ }^{9}$ Eighteenmonth OS and PFS were $81 \%$ and $66 \%$, respectively, a decline from $89 \%$ and $77 \%$ at 12 months. ${ }^{3}$

In the pivotal trial of belantamab, with a median follow-up of 13 months, median PFS and OS in the enrolled population (in an ITT approach) were 2.8 and 13.7 months, respectively. Thirty-one of 97 enrolled patients (32\%) achieved an overall response (Table 1). Twelve-month OS was $57 \% .{ }^{6}$

In the pivotal trials of ide-cel and cilta-cel, the most important adverse events (AEs) included cytokine release syndrome (CRS), neurotoxicity, and thrombocytopenia. CRS of any grade was reported by $84 \%$ and $95 \%$ of patients in the ide-cel and cilta-cel trials, respectively., ${ }^{3,510}$ The median duration of CRS symptoms was 4 to 5 days and were most 


\begin{tabular}{|c|c|c|c|c|c|}
\hline Intervention & Comparator & $\begin{array}{c}\text { Cost per } \\
\text { QALY gained }\end{array}$ & $\begin{array}{l}\text { Cost per } \\
\text { LY gained }\end{array}$ & $\begin{array}{l}\text { Cost per } \\
\text { evLYG }\end{array}$ & $\begin{array}{l}\text { Cost per additional } \\
\text { PFS month gained }\end{array}$ \\
\hline Ide-cel & CAR-T comparator market basket & $\$ 319,000$ & $\$ 250,000$ & $\$ 280,000$ & $\$ 35,000$ \\
\hline Cilta-cel (preliminary) ${ }^{\mathrm{a}}$ & CAR-T comparator market basket & $\$ 253,000$ & $\$ 207,000$ & $\$ 228,000$ & $\$ 17,000$ \\
\hline Belantamab & Belantamab comparator market basket & $\$ 98,000$ & $\$ 70,000$ & $\$ 93,000$ & $\$ 18,000$ \\
\hline
\end{tabular}

likely to be managed with tocilizumab or corticosteroids. In the pivotal ide-cel trial, 3 deaths (2\%) were treatment related (due to CRS, pneumonia, and gastrointestinal hemorrhage) within 8 weeks of infusion, and an additional death from pneumonia was reported within 6 months of infusion. In the pivotal cilta-cel trial, 6 deaths (6\%) were related to treatment (due to sepsis, CRS, lung abscess, respiratory failure, and neurotoxicity).

In the pivotal trial of belantamab, AEs were reported by $98 \%$ of patients, of which $88 \%$ were related to treatment. Nine patients (10\%) discontinued due to AEs. The most important treatment-related AE was keratopathy (a disease of the cornea), reported by $72 \%$ of patients; however, at 13 months follow-up, the majority had recovered. A decline in vision in their better-seeing eye to 20/50 or worse was reported by $18 \%$, but no patients had permanent vision loss. One death (1\%) was treatment related (due to sepsis). ${ }^{6}$

\section{LIMITATIONS OF THE CLINICAL EVIDENCE}

The primary limitations in the clinical evidence include the lack of data from randomized controlled trials and limited follow-up time. The lack of head-to-head comparative data near the time of launch is not unexpected but makes even indirect comparisons needed to guide judgments of relative effectiveness highly uncertain.

Differences in study populations between the single arm trials and in assessing and reporting outcomes further complicate the comparability of the trial results. Although outcomes of ide-cel and cilta-cel show response rates that are better than currently available treatments, there continues to be considerable uncertainty about their long-term efficacy and concerns about potential safety issues. One potential advantage of the CAR-T therapies is the ability for patients to be off treatment for extended periods; however, long-term data are needed to confirm if this holds true over time. The pivotal trial of ide-cel included retreatment, but it is unclear if standard of care for CAR-Ts will include retreatment. For belantamab, the trial data suggest similar response rates to existing therapies, but its use is associated with potentially serious ocular side effects.

\section{LONG-TERM COST-EFFECTIVENESS}

We developed a de novo decision analytic model for the evaluation of treatments for heavily pretreated MM patients. Our model was informed by key clinical trials, previous relevant economic models, as well as by stakeholder input.11,12 Belantamab, cilta-cel, and ide-cel were compared with a triple- or quad-refractory MM comparator market basket that was derived from a contemporaneous observational study of similar patient populations. ${ }^{13}$ For the CAR-T therapies, an initial decision tree was used to calculate the costs and consequences from treatment initiation (ie, leukapheresis) to T-cell infusion.

Outcomes of interest included total life-years (LYs) gained, quality-adjusted life-years (QALYs) gained, equal value life-years gained (evLYG), time spent in progression free state/responding to treatment, and total costs for each intervention over a lifetime time horizon discounted at 3\% per annum. Full details on ICER's cost-effectiveness analysis and model are available on ICER's website at https:// icer.org/wp-content/uploads/2020/10/ICER_MultipleMyeloma_Final-Report_Update_070921.pdf.

The base-case findings suggest that CAR-T therapies provide clinical benefit for this population, with gains in both QALYs and OS over current treatment options. Model findings across all interventions were sensitive to the cost of comparators, PFS and OS estimates, quality of life related to PFS (on and off treatment), and overall health care costs for MM patients.

The incremental cost-effectiveness ratios for ide-cel vs the MM comparator market basket were approximately $\$ 319,000$ per QALY gained, $\$ 250,000$ per LY gained, $\$ 280,000$ per evLYG gained, and $\$ 35,000$ per additional PFS month gained. Incremental cost-effectiveness ratios for cilta-cel vs the MM comparator market basket were updated with newly released 18 -month trial data and found 


\section{TABLE 3 Votes on Other Benefits and Contextual Considerations for Belantamab}

\begin{tabular}{|c|c|c|c|c|c|}
\hline & Major negative & Minor negative & No difference & Minor positive & Major positive \\
\hline $\begin{array}{l}\text { What are the relative effects of belantamab vs usual care on } \\
\text { patients' ability to achieve life goals related to education, } \\
\text { work, or family life? }\end{array}$ & 0 & 1 & 11 & 3 & 0 \\
\hline $\begin{array}{l}\text { What are the relative effects of belantamab vs usual care on } \\
\text { caregivers' quality of life and/or ability to achieve major life } \\
\text { goals relative to education, work, or family life? }\end{array}$ & 0 & 6 & 8 & 1 & 0 \\
\hline $\begin{array}{l}\text { What are the relative effects of belantamab vs usual care on } \\
\text { society's goal of reducing health inequalities? }\end{array}$ & 2 & 5 & 8 & 0 & 0 \\
\hline
\end{tabular}

approximately \$253,000 per QALY gained, $\$ 207,000$ per LY gained, and \$228,000 per evLYG gained.

Base-case findings for belantamab suggest that current list pricing is within commonly cited cost-effectiveness thresholds when compared with a triple-, quad-, or pentarefractory MM market basket. However, small changes in any of the key model inputs changed the belantamab model findings to a significant extent, so our results should be viewed with caution. Table 2 summarizes the full results for all incremental cost-effectiveness outcomes evaluated. A modified societal perspective was explored for all treatments as a scenario analysis and included productivity losses and transportation time to and from health care appointments. Results were not notably different from those of the base-case analysis.

The ICER health benefit price benchmark (HBPB) is a suggested price range based on cost-effectiveness thresholds at the $\$ 100,000$ and $\$ 150,000$ per QALY and per evLYG. The annual HBPB for ide-cel ranged from $\$ 192,000$ to $\$ 265,000$. The annual HBPB for cilta cel ranged from $\$ 230,000$ to $\$ 312,000$. The HBPB for belantamab ranged from $\$ 8,300$ to $\$ 9,500$ per vial. Full results are available on ICER's website at https://icer.org/wp-content/uploads/2020/10/ICER_ Multiple-Myeloma_Final-Report_Update_070921.pdf.

\section{LIMITATIONS OF THE COST-EFFECTIVENESS MODEL}

Considering that evidence was abstracted from single-arm studies, it was difficult to obtain data for a suitable comparator, and significant uncertainty remains regarding how these treatments would compare head-to-head to current best usual care. Furthermore, given that the treatment landscape changes dramatically over short time periods in RRMM and the lack of a quantitative indirect treatment comparison to inform these analyses, caution should be used when interpreting cost-effectiveness estimates, particularly for the cilta-cel results.

\section{Policy Discussion}

The Midwest Comparative Effectiveness Public Advisory Council (CEPAC) is one of the independent appraisal committees convened by ICER to engage in the public deliberation of the evidence on clinical and cost-effectiveness of health care interventions. The Midwest CEPAC is composed of medical evidence experts, including practicing clinicians, methodologists, and leaders in patient engagement and advocacy. Their deliberation includes input from clinical experts and patient representatives specific to the condition under review, as well as formal comment from manufacturers and the public. A policy roundtable concludes each meeting during which representatives from insurers and manufacturers join clinical experts and patient representatives to discuss how best to apply the findings of the evidence to clinical practice, insurance coverage, and pricing negotiations.

The ICER report on treatments for heavily pretreated RRMM was the subject of a Midwest CEPAC meeting on April 16, 2021. Following the discussion, the Midwest CEPAC panel members deliberated on key questions raised by ICER's report. The results of their votes on the clinical evidence were as follows: (1) the panel voted 10-5 that the evidence was not adequate to demonstrate that the net health benefit of belantamab mafodotin is superior to that provided by usual care; (2) the panel voted 15-0 that the evidence was adequate to demonstrate that the net health benefit of ide-cel is superior to usual care; (3) the panel voted 13-2 that the evidence was adequate to demonstrate that the net health benefit of cilta-cel is superior to usual care; and (4) the panel voted 15-0 that the evidence was not adequate to distinguish the net health benefit of ide-cel from cilta-cel.

The CEPAC panel also voted on "other potential benefits" and "contextual considerations" as part of a process intended to signal to policymakers whether there are 


\section{TABLE 4 Votes on Other Benefits and Contextual Considerations for Ide-Cel and Cilta-Cel}

\begin{tabular}{l|c|c|c|c|c}
\hline \multicolumn{1}{c}{ What are the relative effects of ide-cel and cilta-cel vs usual care on the following outcomes? } \\
\hline & $\begin{array}{c}\text { Major negative } \\
\text { effect }\end{array}$ & $\begin{array}{c}\text { Minor negative } \\
\text { effect }\end{array}$ & $\begin{array}{c}\text { No difference } \\
\begin{array}{c}\text { Minor positive } \\
\text { effect }\end{array}\end{array}$ & $\begin{array}{c}\text { Major positive } \\
\text { effect }\end{array}$ \\
\hline $\begin{array}{l}\text { Patients' ability to achieve major life goals related to } \\
\text { education, work, or family life }\end{array}$ & 0 & 0 & 1 & 8 \\
\hline $\begin{array}{l}\text { Caregivers' quality of life and/or ability to achieve major life } \\
\text { goals related to education, work, or family life }\end{array}$ & 0 & 0 & 0 & 13 \\
\hline $\begin{array}{l}\text { Patients' ability to manage and sustain treatment given the } \\
\text { complexity of regimen }\end{array}$ & 0 & 0 & 1 & 12 \\
\hline \begin{tabular}{l} 
Society's goal of reducing health inequities \\
\hline
\end{tabular}
\end{tabular}

\section{TABLE 5 Votes on Long-Term Value for Money of Belantamab and Ide-Cel}

\begin{tabular}{|c|c|c|c|}
\hline \multicolumn{4}{|c|}{$\begin{array}{c}\text { Given the available evidence on comparative effectiveness and } \\
\text { incremental cost-effectiveness and considering other benefits, } \\
\text { disadvantages, and contextual considerations, what is the } \\
\text { long-term value for money of treatment at current pricing with } \\
\text { belantamab or ide-cel vs usual care? }\end{array}$} \\
\hline & $\begin{array}{l}\text { Low long-term } \\
\text { value for money }\end{array}$ & $\begin{array}{l}\text { Intermediate } \\
\text { long-term value } \\
\text { for money }\end{array}$ & $\begin{array}{l}\text { High long-term } \\
\text { value for money }\end{array}$ \\
\hline Belantamab & 8 & 6 & 1 \\
\hline Ide-cel & 9 & 5 & 0 \\
\hline
\end{tabular}

important considerations when making judgments about long-term value for money not adequately captured in analyses of clinical and/or cost-effectiveness. The results of these votes are shown in Tables 3-5.

Following its deliberation on the evidence, the Midwest CEPAC panel engaged in a moderated discussion with a policy roundtable about how best to apply the evidence on CAR-T therapies and belantamab for TCRMM to policy and practice. The full set of policy recommendations can be found in the Final Evidence Report on the ICER website: https://icer.org/wp-content/uploads/2020/10/ ICER_Multiple-Myeloma_Final-Report_Update_070921. pdf. Several key policy recommendations follow:

- All stakeholders have a responsibility and an important role to play in ensuring that effective new treatment options for patients with MM are introduced in a way that will help reduce health inequities.

o Manufacturers should engage with a variety of people from diverse communities to help inform the design and implementation of clinical trials and to ensure that people of color and those from less advantaged backgrounds are adequately represented in the trials. In addition, manufacturers should moderate new treatment pricing. Even with insurance coverage, cost is a tremendous driver of health inequities.

o Payers should recognize that, in addition to often steep out-of-pocket costs for the treatments, there are often ancillary costs that can become real barriers to care and exacerbate inequities. Payers should develop coverage that creates a broader package of benefits so that patients who face financial or logistical hurdles can have equal access to specialized care at Centers of Excellence, if desired.

o Clinicians and clinical societies should conduct (or continue to conduct) active outreach and education to underserved communities and the general oncologists and other members of the health care team serving those communities to get new, effective treatments to those patients who would benefit most.

- The clinical research community should move rapidly to address key gaps in evidence for treatments for MM. These gaps include whether patients can stop therapy while in response (which could decrease side effects for patients and decrease costs for payers), how well the clinical trial populations reflect the target populations for treatment, data on preferences and patient-reported outcomes in historically disadvantaged populations, and the clinical characteristics of the disease and its affected populations that may be predictive of response.

- Manufacturers should seek to set prices that will foster affordability and good access for all patients by aligning prices with the patient-centered therapeutic value of their treatments. In the setting of these new interventions for MM, while there is considerable hope associated with the promise of the therapies, there also remains substantial uncertainty regarding their longer-term safety and effectiveness, and the platform on which they are based 
has been funded in part with taxpayer money. Manufacturer pricing should also reflect these considerations in moderating launch pricing.

- Payers should use the FDA label as the guide to coverage policy and engage clinical experts and diverse patient representatives in considering how to address coverage issues for which there is limited or no evidence at the current time.

\section{DISCLOSURES}

Funding for this summary was contributed by Arnold Ventures, California Health Care Foundation, The Donaghue Foundation, Harvard Pilgrim Health Care, and Kaiser Foundation Health Plan to the Institute for Clinical and Economic Review (ICER), an independent organization that evaluates the evidence on the value of health care interventions.

ICER's annual policy summit is supported by dues from AbbVie, Aetna, America's Health Insurance Plans, Anthem, Alnylam, AstraZeneca, Biogen, Blue Shield of CA, Boehringer-Ingelheim, Cambia Health Services, CVS, Editas, Evolve Pharmacy, Express Scripts, Genentech/Roche, GlaxoSmithKline, Harvard Pilgrim, Health Care Service Corporation, HealthFirst, Health Partners, Humana, Johnson \& Johnson (Janssen), Kaiser Permanente, LEO Pharma, Mallinckrodt, Merck, Novartis, National Pharmaceutical Council, Pfizer Premera, Prime Therapeutics, Regeneron, Sanofi, Spark Therapeutics, uniQure, and United Healthcare.

Beinfeld, Fluetsch, and Pearson are employed by ICER. Ollendorf received funding from ICER for work on this summary and reports consulting and other personal fees from EMD Serono, Amgen, Analysis Group, Aspen Institute/ University of Southern California, GalbraithWight, Cytokinetics, Executive Insight, Sunovion, University of Colorado, World Health Organization, and Eli Lilly, unrelated to this work. Lee and McQueen received funding from ICER for work on this summary.

\section{ACKNOWLEDGMENTS}

The authors thank Mel Wittington, Eric Gutierrez, Sue Kwon, Belen Herce-Hagiwara, Liis Shea, and Monica Fredrick for their contributions to this report.

\section{REFERENCES}

1. Mikhael J. Treatment options for tripleclass refractory multiple myeloma. Clin Lymphoma Myeloma Leuk. 2020;20(1):1-7.

2. National Cancer Institute. Cancer stat facts: myeloma. Accessed July 24, 2021. https://seer.cancer.gov/statfacts/html/ mulmy.html

3. Berdeja JG, Madduri D, Usmani SZ, et al. Ciltacabtagene autoleucel, a B-cell maturation antigen-directed chimeric antigen receptor T-cell therapy in patients with relapsed or refractory multiple myeloma (CARTITUDE-1): a phase 1b/2 open-label study. Lancet. 398(10297):314-24. doi: 10.1016/S0140-6736(21)00933-8

4. Rajkumar SV. Multiple myeloma: 2020 update on diagnosis, risk-stratification and management. Am J Hematol. 2020;95(5):548-67.

5. Munshi NC, Anderson LD Jr., Shah N, et al. Idecabtagene vicleucel in relapsed and refractory multiple myeloma. N Engl J Med. 2021;384(8):705-16.

6. Lonial S, Lee HC, Badros A, et al. Belantamab mafodotin for relapsed or refractory multiple myeloma (DREAMM-2): a two-arm, randomised, open-label, phase 2 study. Lancet Oncol. 2020;21(2):207-21.

7. Celgene. Efficacy and safety study of bb2121 in subjects with relapsed and refractory multiple myeloma (KarMMa). ClinicalTrials.gov Identifier: NCT03361748. 2020. Accessed. https://clinicaltrials.gov/ ct2/show/record/NCT03361748
8. Harris J, Dobkowski D. Durable responses observed at 18 months with cilta-cel to treat relapsed/refractory multiple myeloma. Cancer Network. June 8, 2021. Accessed June 24, 2021. https://www.cancernetwork.com/ view/durable-responses-observedat-18-months-with-cilta-cel-to-treatrelapsed-refractory-multiple-myeloma

9. Janssen Research \& Development. A study of JNJ-68284528, a chimeric antigen receptor $\mathrm{T}$ cell (CAR-T) therapy directed against $\mathrm{B}$-cell maturation antigen (BCMA) in participants with relapsed or refractory multiple myeloma (CARTITUDE-1). ClinicalTrials.gov Identifier: NCT03548207. 2020. Accessed August 5, 2021. https://www.clinicaltrials.gov/ct2/ show/NCT03548207

10. Munshi NC, Anderson JLD, Shah N, et al. 8503: Idecabtagene vicleucel (idecel; bb2121), a BCMA-targeted CAR T-cell therapy, in patients with relapsed and refractory multiple myeloma (RRMM): initial KarMMa results [abstract]. J Clin Oncol. 2020;39(15 Suppl):8503 doi: 10.1200/JCO.2020.38.15_suppl.8503

11. Asrar MM, Lad DP, Prinja S, Bansal D. A systematic review of economic evaluations of treatment regimens in multiple myeloma. Expert Rev Pharmacoecon Outcomes Res. June 27, 2020. Online ahead of print. Accessed August 5, 2021. https:// doi.org/10.1080/14737167.2020.1779064.

12. Carlson JJ, Guzauskas GF, Chapman $\mathrm{RH}$, et al. Cost-effectiveness of drugs to treat relapsed/refractory multiple myeloma in the United States. J Manag Care Spec Pharm. 2018;24(1):29-38. doi: 10.18553/jmcp.2018.24.1.29

13. Gandhi UH, Cornell RF, Lakshman A, et al. Outcomes of patients with multiple myeloma refractory to CD38-targeted monoclonal antibody therapy. Leukemia. 2019;33(9):2266-75. 